\title{
Hidden Markov model-based approach for generation of Pitman shorthand language symbols for consonants and vowels from spoken English
}

\author{
G HEMANTHA KUMAR, M RAVISHANKAR, P NAGABUSHAN \\ and BASAVARAJ S ANAMI \\ Dept. of Studies in Computer Science, University of Mysore, Mysore 570 006, \\ India \\ e-mail: ravishankarmc@mailcity.com
}

MS received 3 December 2004; revised 2 March 2006

\begin{abstract}
Pitman shorthand language (PSL) is a widely practised medium for transcribing/recording speech to text (StT) in English. This recording medium continues to exist in spite of considerable development in speech processing systems (SPS), because of its ability to record spoken/dictated text at high speeds of more than 120 words per minute. Hence, scope exists for exploiting this potential of PSL in present SPS.

In this paper, an approach for feature extraction using Mel frequency cepstral coefficients (MFCC) and classification using hidden Markov models (HMM) for generating strokes comprising consonants and vowels (CV) in the process of production of Pitman shorthand language from spoken English is proposed. The proposed method is tested on a large number of samples, drawn from different speakers and the results are encouraging. The work is useful in total automation of PSL processing.
\end{abstract}

Keywords. Mel frequency cepstral coefficient (MFCC); hidden Markov model (HMM); speech processing; script generation; Pitman shorthand language.

\section{Introduction}

Speech is one of the most basic means of human communication. Exchange of ideas among human beings is carried out with the aid of communication and has facilitated the development of technology in various forms that we are so accustomed to. Much benefit could be gained if people could communicate with various man-made gadgets. Investigations in this field have led to the development of automatic speech recognition systems.

Production of speech can be briefly explained with the help of the human vocal physiological mechanism. Speech is produced by vocal organs, the main source of sound production being the lungs with the diaphragm. While speaking, air is forced through the glottis between the vocal chords and the larynx to the three main cavities of the vocal tract: the pharynx, and the oral and nasal cavities, from where the air exits through the nose and mouth respectively. 


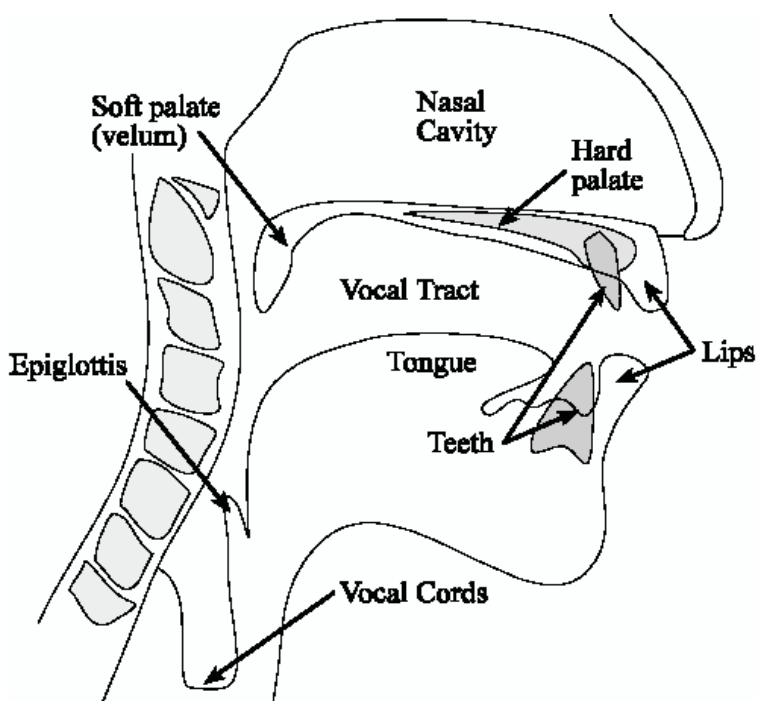

Figure 1. Vocal organs in human beings.

The ' $v$ ' shaped opening between the glottises is the most important source of sound in the vocal system. The vocal chords may act in several different ways in speech processing, the most important function being the modulation of the airflow by rapidly opening and closing the cavity causing the buzzing sound from which vowels and consonants are produced. The vocal organs in human beings are shown in figure 1.

Signal level processing of input speech for its recognition is fairly reported in the literature (Davis et al 1952; Rabiner et al 1979). But no work pertaining to generation of Pitman shorthand language symbols for a combination of consonants and vowels $(\mathrm{CV})$ corresponding to the words of spoken English is reported in the literature. This is the motivation for the present work.

The proposed approach is developed in two distinct phases. The first phase is referred to as the training phase and the second as the testing phase. In the training phase, speech samples of consonant-vowel $(\mathrm{CV})$ combination are provided as input to induce the system to build a reference model for later recognition. During the testing phase, the unknown inputs of the consonant-vowel combination are matched with those of the stored reference model and the inputs are recognized.

The remaining part of the paper is organized into five sections. Overview of PSL is given in $\S 2$, the proposed method is presented in $\S 3$, Overall approach and the developed algorithm is proposed in $\S 4$, Experimental setup is given in $\S 5$, Results of recognition of the consonantvowel $(\mathrm{CV})$ combination are discussed in $\S 6$ and the overall work is concluded in $\S 7$.

\section{Overview of consonants and vowels in Pitman shorthand language}

The character set of PSL consists of consonants, delimiters and other symbols, which are shown in table 1 . The consonants are the result of audible friction or stopping of breathe in some part of the mouth or throat (Pitman 1976). There are 26 consonant symbols in shorthand. These symbols can be thin or thick, according to which the symbols represent different phonetic characters. The vowels are the result of keeping the mouth passage so open as to not cause any audible friction and thus passing of voiced breathe through it. There are six long 
Table 1. PSL basic strokes and English consonants.

\begin{tabular}{|c|c|c|c|}
\hline Character & Phonetic name & Stroke nature & $\begin{array}{l}\text { English consonant } \\
\text { equivalent }\end{array}$ \\
\hline \multicolumn{4}{|l|}{ Explodents } \\
\hline 1 & Pee & (thin) & $\mathrm{P}$ \\
\hline 1 & Bee & (thick) & B \\
\hline & Tee & (thin) & $\mathrm{T}$ \\
\hline 1 & Dee & (thick) & $\mathrm{D}$ \\
\hline / & Chay & (thin) & $\mathrm{CH}$ \\
\hline / & Jay & (thick) & $\mathrm{J}$ \\
\hline & Kay & (thin) & $\mathrm{K}$ \\
\hline & Gay & (thick) & $\mathrm{G}$ \\
\hline \multicolumn{4}{|c|}{ Continuants } \\
\hline & Ef & (thin) & $\mathrm{F}$ \\
\hline & Vee & (thick) & $\mathrm{V}$ \\
\hline( & Ith & (thin) & $\mathrm{TH}$ \\
\hline ( & Thee & (thick) & $\mathrm{TH}$ \\
\hline ） & Ess & (thin) & S \\
\hline ) & Zee & (thick) & $\mathrm{Z}$ \\
\hline & Ish & (thin) & $\mathrm{SH}$ \\
\hline & Zhee & (thick) & $\mathrm{ZH}$ \\
\hline \multicolumn{4}{|l|}{ Nasals } \\
\hline & Em & (thin) & M \\
\hline$\cup$ & En & (thin) & $\mathrm{N}$ \\
\hline$\cap$ & Ing & (thick) & NG \\
\hline 1 & Ar, ray & (thin) & $\mathrm{R}$ \\
\hline & El & (thin) & $\mathrm{L}$ \\
\hline \multicolumn{4}{|c|}{ Coalescents } \\
\hline & Way & (thin) & W \\
\hline & Yay & (thin) & $\mathrm{Y}$ \\
\hline \multicolumn{4}{|l|}{ Aspirates } \\
\hline & Hay & (thin) & $\mathrm{H}$ \\
\hline$\sigma$ & Hay & (thin) & $\mathrm{H}$ \\
\hline
\end{tabular}


vowel sounds in PSL as heard in the words like wah!, ale, each, all,oak,ooze,etc and six short vowel sounds as heard in the words like at, etch, it, odd, tub, book etc.

This work comprises generation of symbols of basic set of consonants, short vowels, long vowels, grammalogues, diphthongs and punctuation. The first eight consonants, represented by straight strokes, are called "explodents", because in pronouncing them, the outgoing breath is forced out in a sudden gust through barriers previously closed.

The next eight consonants, represented by upright or sloping curves, are called "continuants", because in uttering these, the outgoing breath, instead of being expelled suddenly, is allowed to escape in a continuous stream through similar barriers that are partially open. Closing the successive barriers in the mouth against the outgoing air-stream produces the "nasals", represented by horizontal curves, so that it escapes through the nose (Pitman 1976). The "liquids" flow into union with other consonants, and thus make double consonants, as in the words, cliff, dry, etc, where the 1 or $\mathrm{r}$ blends with the preceding consonant. The "coalescent" precede vowels and coalesce or unite with them. The "aspirate" is breathing upon a following vowel. Thus, by breathing upon the vowel "a" in the word "at", the word is changed to "hat". The language supports other features like grammalogues, diphthongs, triphones, and phraseograms etc, which are again formed out of these strokes.

The vowels also appear before or after the strokes depending on whether the vowels precede or succeed in the utterance of a word. The vowels are represented by two types of symbols namely dash (-) and dot (•). The vowels can also be thick or thin, similar to consonants. Every stroke in PSL is formed out of different combinations of stroke types and vowels. The vowels also appear before or after the strokes depending on whether the vowels precede or succeed in the utterance of a word. The vowels can also be thick or thin, similar to consonants. Every stroke in PSL is formed out of different combinations of stroke types and vowels. There are totally 624 combinations ( 26 strokes and 24 vowel positions). The three positions for vowels, which override the strokes, namely first, second and third, are illustrated in figure 2. The first three and second three long vowels are represented by thick dots and thick dashes respectively. The same convention is also followed for short vowels as well, except that thin dots and dashes are used respectively. The vowel-signs are put in the places which correspond with their numbers. A heavy dot in the first-place represents the long vowel $a h$; in the second place it represents the long vowel $a$; in the third place it represents the long vowel $e$. A heavy dash in the first place represents the long vowel $a w$; in the second place it represents the long vowel $o$; in the third place it represents the long vowel $o o$.

\subsection{Recording the dictated/spoken text}

When the speech recording method is adopted, there are various problems that one needs to tackle. An on-line recording medium is required, and reproducing this recorded speech into English text is still an open research problem in the area of speech recognition. Noises around the premises of the location of recording may affect the reproduction of the dictated speech. Pronunciation varies from individual to individual, and so does the accent. This poses,

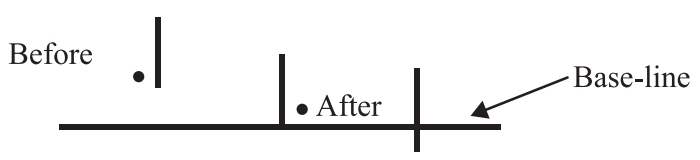

(1)

(2)

Figure 2. Three positions for the strokes. 


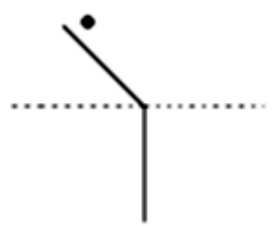

(a)

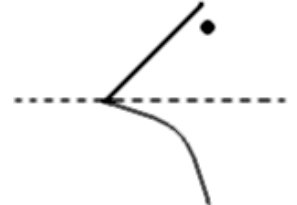

(b)
Figure 3. An example of a stroke for the word (a) 'BAT' (b) 'JAR'.

probably, the most difficult problem in speech recognition, even to a human being, let alone a computer system. Another problem is in recognizing words that are homophones, which are words that have the same phonetic spelling, but differ in their linguistic spellings.

In Pitman shorthand, a stroke consists of straight lines/arcs of a circle/straight line with hooks (either dark or light) which represent consonants. The different stroke types are shown in table 1. A stroke is a concatenation of these primitive symbols, called segments. Examples of strokes are as shown in figure 3. Each segment has a standard length of $1 / 3 \mathrm{rd}$ of an inch. This corresponds to a length of around 18 pixels on our system (VGA monitor of $640 \times 480$ resolution). A consonant symbol may be associated with an overriding dot or a dash, which represents the vowel sounds. A dot or a dash can appear either below or above or to the left or right of the consonant symbol in any of the three fragments. In our system, each fragment is of 6 pixels length. The work in this paper pertains to automation of writing the PSL equivalent strokes for English language words comprising consonants and vowels, which will be helpful in generation of the complete PSL text for spoken English sentences.

\section{Proposed methodology}

The proposed methodology is divided into two stages, which is shown in figure 4 . The first stage involves feature extraction wherein short temporal or spectral parameters of speech are extracted using Mel frequency cepstral coefficients (MFCC). The second stage involves classification of speech using hidden markov models (HMM), wherein the obtained parameters are compared with reference parameters and a decision is made based on the minimum distortion rule (Rabiner \& Juang 2003).

\subsection{Feature extraction}

The proposed approach for feature extraction is given in figure 5. The voice input is recorded using the microphone and sound recorder utility supported by Windows 98 . The speech signal comprises both voiced and unvoiced signals. For effective speech to text conversion, it is necessary to extract only the voiced part of the speech signal and further normalize it. Typically, speech analysis is done using a fixed length frame, or an analysis window. In this work, the speech is sampled at $16 \mathrm{kHz}$ and subjected to low-pass filtering with a corner frequency lower than $8 \mathrm{kHz}$ to prevent spectral aliasing. The signal obtained is enframed for later processing.

3.1a Windowing: Each individual frame of a speech signal is windowed so as to minimize the signal discontinuities at the beginning and at the end of each frame and thus the spectral distortion is minimized. The window is defined as given below.

$$
w(n) ; \quad \text { where } \quad 0 \leq n \leq(N-1) .
$$




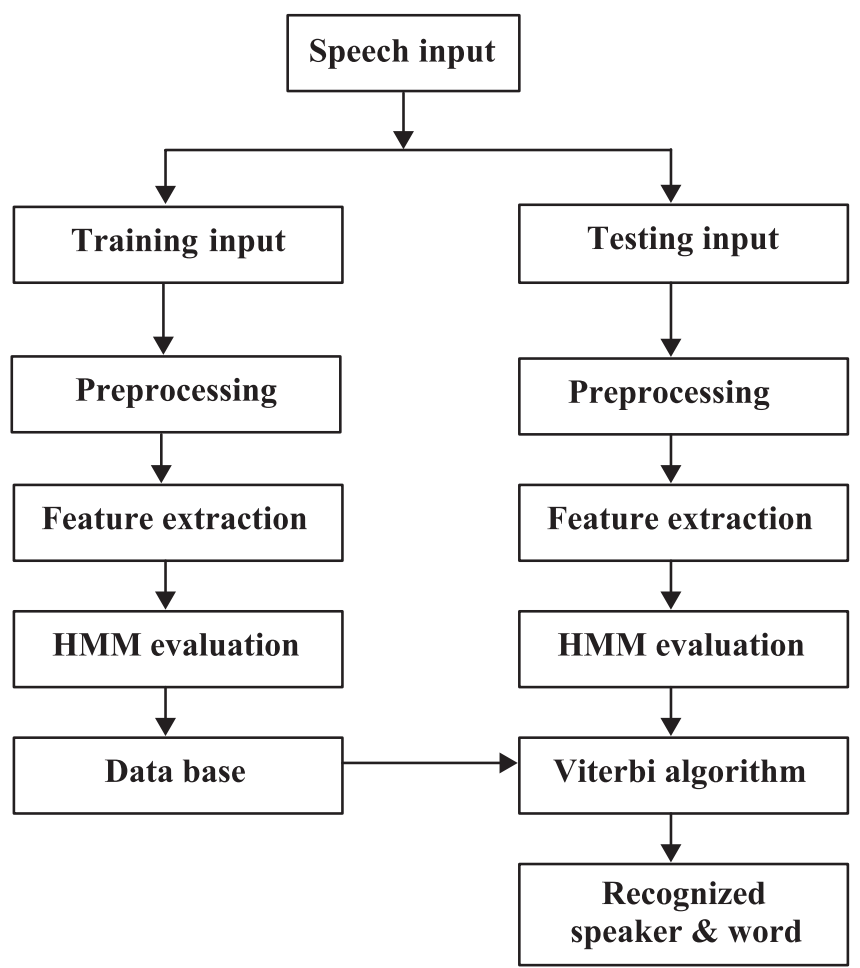

Figure 4. Overall block diagram of proposed method.

$N$ is the number of samples in each frame. The result of windowing is the signal $(y(n))$ and is defined as

$$
y(n)=x(n) w(n), \quad \text { where } 0 \leq n \leq(N-1) .
$$

The Hamming window, $w(n)$, used in the work is given by

$$
w(n)=0.54-0.46 \cos [2 \pi n /(N-1)] \quad \text { and } \quad 0 \leq n \leq(N-1) .
$$

The purpose of the window is to favour samples towards the centre of the window. This characteristic coupled with the overlapping attempts to smoothen the varying parameters. Fast Fourier Transform is applied to the windowed samples, which converts each frame of $N$ samples from the time domain into the frequency domain.

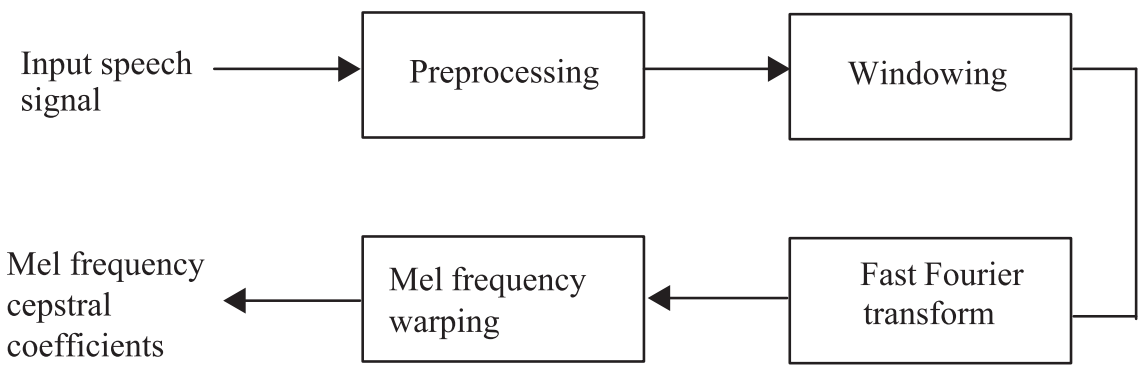

Figure 5. Schematic block diagram illustrating feature extraction. 
3.1b Fast fourier transform (FFT): The FFT is defined on the set of $N$ samples $\left\{X_{n}\right\}$ as,

$$
X_{n}=\sum_{k=0}^{N-1} x_{k} e^{-2 \pi k n / N}, \quad \text { where } \quad n=0,1,2 \ldots N-1
$$

In general, $X_{n} \mathrm{~s}$ are the complex numbers. The resulting sequence of $X_{n} \mathrm{~s}$ is interpreted as given below:

(i) When $(n=0)$, it corresponds to zero frequency.

(ii) When $1 \leq n \leq(N / 2-1)$, it corresponds to positive frequencies $\left(0<f<F_{S} / 2\right)$.

(iii) When $N / 2+1 \leq n \leq N-1$, it corresponds to negative frequencies $\left(-F_{s} / 2<f<0\right)$.

Here, $F s$ denote the sampling frequencies. The result so obtained is often referred to as 'spectrum' or 'periodogram'. It is known that human perception of the frequency contents of sounds for speech signals do not follow a linear scale. Hence, for each tone with an actual frequency ' $f$ ', measured in $\mathrm{Hz}$, a subjective pitch is measured on a scale called 'Mel-frequency scale'.

3.1c Mel frequency warping: The Mel frequency scale is the linear frequency spacing below $1000 \mathrm{~Hz}$ and a logarithmic spacing above $1000 \mathrm{~Hz}$. The following approximate empirical relationship to compute the Mel frequencies (also called Mel's) for a given frequency $f$ expressed in $\mathrm{Hz}$ is as given below.

$$
\operatorname{Mel}(f)=2595 \times \log (1+f / 700)
$$

To simulate the subjective spectrum, a filter bank spaced uniformly on the Mel scale is used, which is shown in figure 6 . This filter bank has a triangular bandpass frequency response and the spacing. The bandwidth is determined by a constant Mel frequency interval. This filter bank is applied in the frequency domain and amounts to taking only triangle-shape windows on the spectrum (Vergin et al 1999).

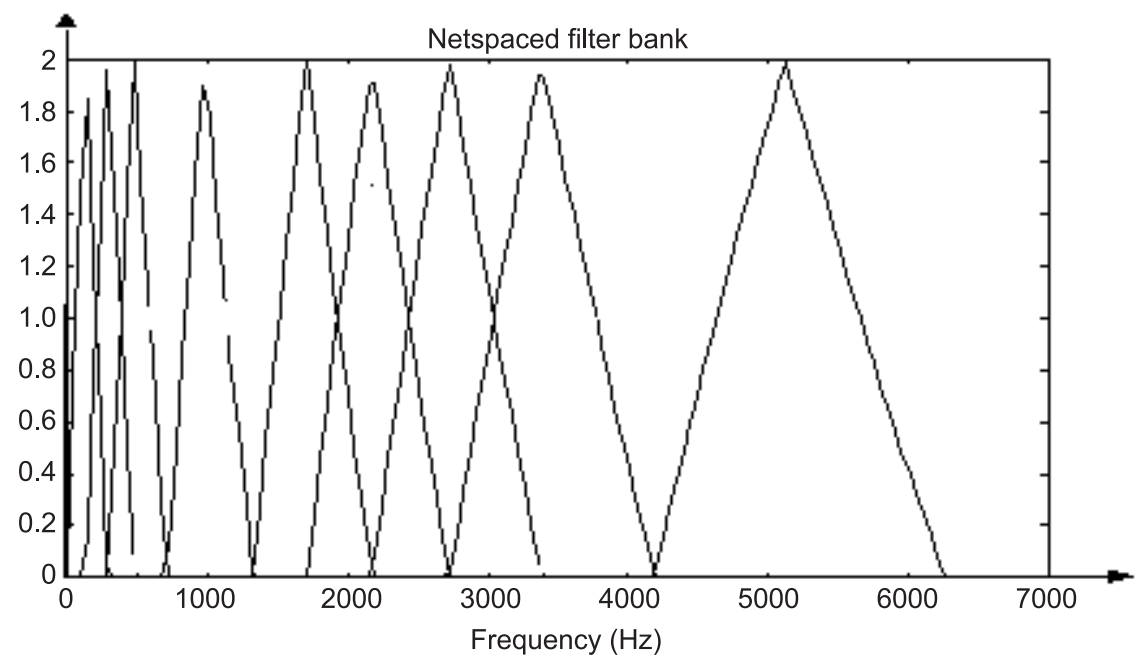

Figure 6. Mel triangular filter bank. 


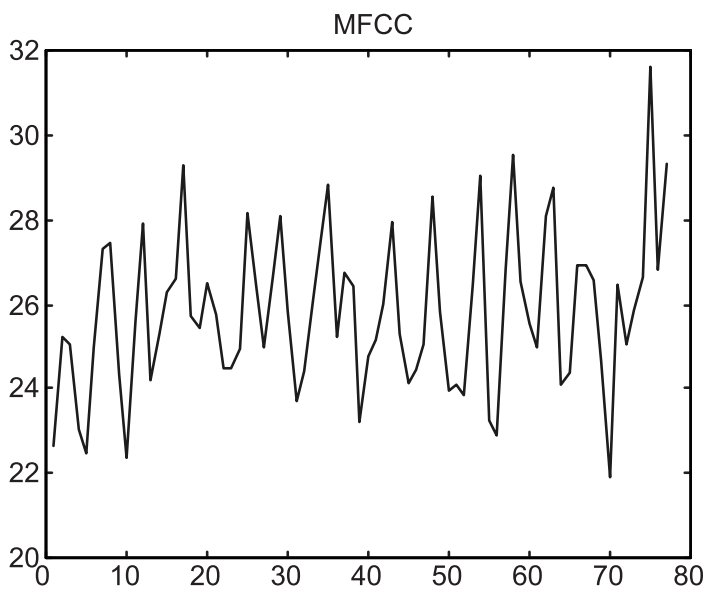

Figure 7. Mel frequency cepstral coefficient (MFCC) plot for the syllable ' $\mathrm{Pa}$ '.

Further, the log Mel frequency spectrum is converted back to time domain using Discrete Cosine Transform (DCT). The resultant is called the Mel Frequency Cepstrum Coefficients (MFCC) and are calculated using,

$$
c_{n}=\sum_{k=1}^{K}\left(\log S_{k}\right) \cos \left[n\left(k-\frac{1}{2}\right) \frac{\pi}{k}\right] .
$$

Thus, for each speech frame, a set of Mel frequency cepstrum coefficients is computed, which is plotted as shown in figure 7. These sets of cepstral coefficients are stored in the codebook as a reference model. Furthermore, feature matching process involves comparison of extracted features with stored reference models.

\subsection{Classification}

The hidden Markov model (HMM) is a well known and widely used statistical method for characterizing the spectral properties of the frames of a pattern. The underlying assumption of the HMM is that the speech signal is well characterized as a parametric random process and the parameters of the stochastic process are determined in a precise and well-defined manner. In the HMM statistical approach, speech is represented according to some probability distributions. In the Markov models, if the observation is a probabilistic function of the state, it is called a hidden Markov model because it has a doubly embedded stochastic process with an underlying stochastic process that is not directly observable (it is hidden) but can be observed only through another set of stochastic processes that produce the sequence of observations (Rabiner \& Juang 2003).

An HMM for discrete symbol observations is characterized by the following elements:

- Number of states $(N)$

- Number of observation symbols $(M)$

- State transition probability $(A)$

- Observation symbol probability $(B)$

- Initial state probability (П) 


\section{$a_{1 .}$ : transition probabillty from state $S_{i}$ to $S_{j}$}

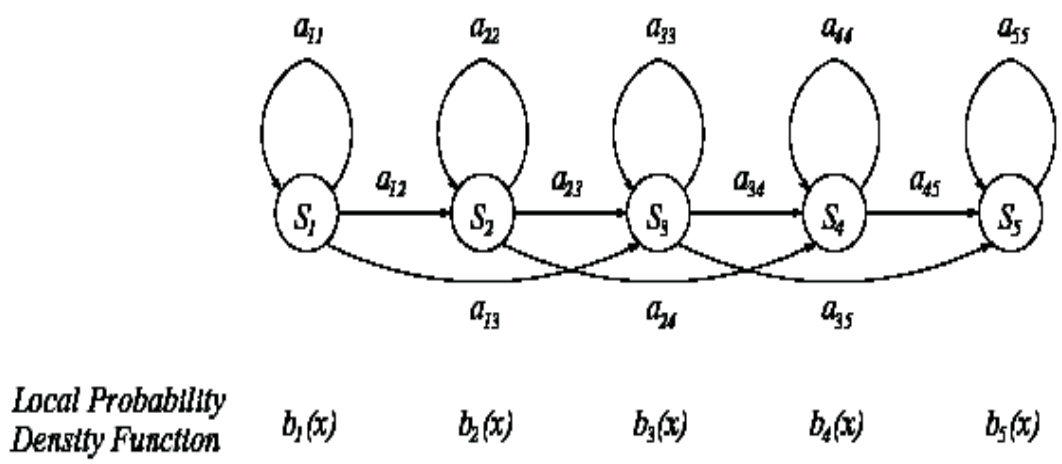

Figure 8. A left to right hidden Markov model with five states.

3.2a Number of states $(N)$ : Although the states are hidden, for any practical application there is often some physical significance attached to the states or the sets of states of the model. Generally the states as given in figure 8 are interconnected in such a way that any state is reached from any other state.

3.2b Number of observation symbols $(M)$ : The observation symbols correspond to the physical output of the system being modelled. The number of distinct observation symbols are denoted as

$$
V=\left\{v_{1}, v_{2}, v_{3} \ldots v_{m}\right\}
$$

3.2c State transition probability $(A):$ State transition probability distribution

$$
A=\left\{a_{i j}\right\}
$$

where

$$
a_{i j}=P\left[q_{t+1}=j / q_{t}=i\right], \quad \text { and } \quad 1 \leq i, j \leq M .
$$

for a special case in which any other state can be reached in a single step. We have $a_{i j} \geq 0$ for all values of $i, j$.

3.2d Observation symbol probability $(B)$ : Observation symbol probability distribution

$$
B=\{b j(k)\},
$$

where

$$
b_{j}(k)=P\left[O_{t}=v_{t} / q_{t}=j\right], \quad \text { and } \quad 1<k<M,
$$

defines the symbol distribution in state $j$, for $j=1,2,3,4 \ldots N$.

3.2e Initial state distribution $(\Pi)$ : Initial state distribution $\Pi$ is given by

$$
\Pi=\left\{\Pi_{i}\right\}, \quad \Pi_{i}=P\left[q_{t}=i\right] .
$$


Therefore, it is seen that a complete specification of an HMM requires specification of two model parameters, $N$ and $M$, observation symbols and the three sets of probability measures $A, B$ and $\Pi$. For convenience, the complete parameter set of an HMM model is denoted by

$$
\lambda=(A, B, \Pi) .
$$

The parameter $\lambda$ defines a probability measure for the observation sequence $O$, i.e., $P(O / \lambda)$. The observation sequence is nothing but the feature analysis (MFCC) of the speech corresponding to the word. Given two HMMs $\lambda 1$ and $\lambda 2$, we have determined a reasonable measure of the similarity using the concept of logarithmic distance by defining the distance measure $D(\lambda 1, \lambda 2)$ between two Markov models $\lambda 1$ and $\lambda 2$, as given in

$$
D\left(\lambda_{1}, \lambda_{2}\right)=1 / T\left[\log _{10} P\left(O_{2} / \lambda_{1}\right)-\log _{10} P\left(O_{2} / \lambda_{2}\right)\right],
$$

where $O_{2}=\left(O_{1}, O_{2} \ldots O_{t}\right)$ is a sequence of observations generated by the model $\lambda_{2}$. Basically the above expression for $O_{2}$ is the measure of how well model $\lambda_{1}$ matches observations generated by model $\lambda_{2}$ the matched observations generated by it. The symmetrized version is given by

$$
D\left(\lambda_{1}, \lambda_{2}\right)=\left(D\left(\lambda_{1}, \lambda_{2}\right)+D\left(\lambda_{1}, \lambda_{2}\right)\right) / 2 .
$$

Vocabulary of words to be recognized is modelled by a distinct HMM. Each word in the vocabulary has a training set of $k$ utterances by different speakers (Rabiner \& Huang 2003). Each utterance constitutes an observation sequence of MFCC. Isolated word speech recognition consists of the following two major steps.

(1) For each word in the vocabulary build an HMM and estimate the model parameters $(A, B, \Pi)$, which represent the likelihood of the training set observation vectors.

(2) For each unknown word to be recognized, measurements of the observation sequence via feature analysis of the speech corresponding to the word, are made. Finally the word is selected using the Viterbi algorithm whose model likelihood is maximum as given in figure 9 .

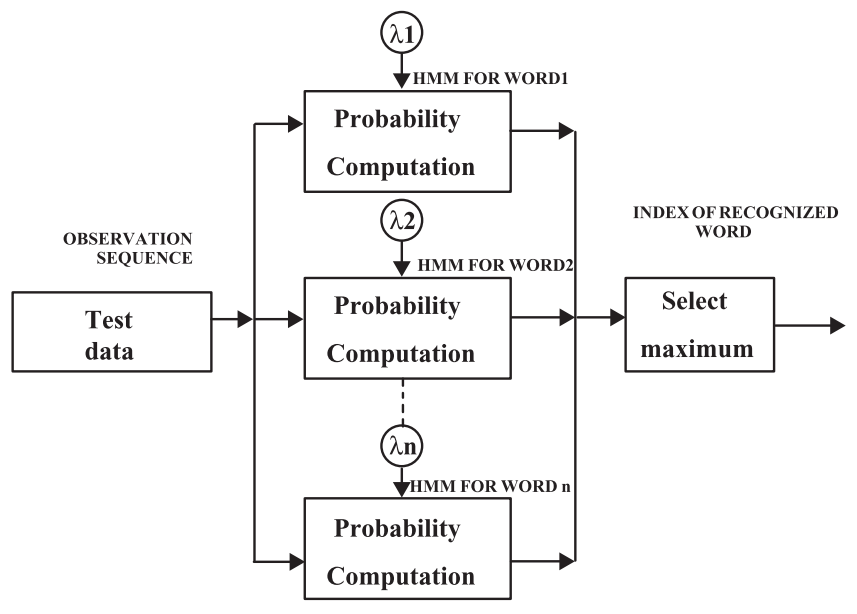

Figure 9. Block diagram of HMM word recognizer. 
An HMM is formed for both training and testing phases by evaluating the model parameters $A, B$ and $\Pi$. A word is recognized after comparing the testing model with the help of the Viterbi algorithm

3.2f Viterbi algorithm: The Viterbi algorithm is used to find the single best state sequence for the given observation sequence (Rabiner \& Huang 2003). The complete procedure for finding the best state sequence includes the following steps.

- Initialization

- Recursion

- Termination

- Path back tracking

Forward recursion yields the complete likelihood that is more efficient, which is functionally equivalent to the direct summation of the likelihoods of all possible legal paths within a model. It requires both multiplication and addition of likelihoods and is converted to the logarithmic domain to handle the wide range of values for products. However, there is also the addition that the logarithmic probabilities must be exponentiated. When the complete likelihood is used, it is often useful to find the single best state sequence to explain the observation sequence. This requires modifying the forward recurrence. Hence, the best path or Viterbi approximation is obtained.

\section{Overall approach and algorithm}

The present work is an attempt towards generation of PSL symbols for consonant-vowel combinations (CV) from spoken/uttered English words, leading to the further development of a complete system for automatic production of PSL text from spoken English text. The overall approach is divided into two phases.

In phase 1 sampling of speech signal, identification of start and end points, enframing of the signal and computation of MFCC are carried out. Since we are using HMM for classification, the MFCC's are considered as the observation sequence for a particular spoken consonants and vowels combination. The spectral coefficients are used to calculate the mean and the covariance. These are then considered the observation sequence vectors used for building an HMM. To build a hidden Markov model for each consonant and vowel combination, we have to compute state transition probability $(A)$, observation symbol probability $(B)$ and initial state distribution $(\Pi)$ as stated earlier. The consonant and vowel combination spoken by each speaker is passed through this routine, evaluating the model parameters. This procedure is repeated for the other speakers. In this work we use the left-right HMM, which has the desired property of modelling signals whose properties change over time in a successive manner.

In phase 2, the same procedure is followed till evaluating the HMM, which means that the preprocessing of the input speech signal, feature extraction and building a HMM are carried out. The observation sequence and the HMM formed by the test input are compared with all models present in the database and the best match is then considered as the recognized word. For this observation, sequence of the test input along with $\lambda$ is passed to the Viterbi algorithm to calculate the logarithmic distance between the test and the training data. The Viterbi algorithm helps in computing the logarithmic distance and is also used for path backtracking. The decision on the recognized CV combinations is taken based on the maximum logarithmic likelihood of the test data matching one of the trained data. The entire process is put forth as an algorithm. 
Algorithm: CV-to-PSL text

Input: $\quad$ Spoken English consonant-vowel combinations

Output: Written Pitman shorthand language equivalent of English consonant-vowel combination.

Begin

Step 1: Call Phase-1

Step 2: $\quad$ Call Phase-2

End

Phase - 1: Training

Begin

Step 1: Input speech signal is sampled.

Step 2: Start and end points of speech signal are detected.

Step 3: The speech signal is enframed.

Step 4: Enframed speech signal is windowed by using Hamming Window.

Step 5: Fast Fourier transform is applied to the windowed signal.

Step 6: Mel frequency cepstral coefficients (MFCC) are calculated.

Step 7: $\quad$ HMM is built for each consonant-vowel, i.e $\lambda(A, B, \Pi)$ is evaluated and stored in the database.

End

Phase - 2: Recognition

Begin

Step 1: Input speech signal is sampled.

Step 2: Start and end points of speech signal are detected.

Step 3: The speech signal is enframed.

Step 4: Enframed speech signal is windowed by using Hamming Window.

Step 5: Fast Fourier transform is applied to the windowed signal.

Step 6: Mel frequency cepstral coefficients (MFCC) are calculated.

Step 7: $\quad$ HMM is built for each consonant-vowel, i.e $\lambda(A, B, \Pi)$ is evaluated.

Step 8: Observation sequence and the HMM formed by the test input are compared with all models present in the database using Viterbi algorithm.

Step 9: The decision on the recognized word is taken based on the maximum logarithmic likelihood of the test data matching with one of the trained data.

End

\section{Experimental setup}

A total of 500 speakers comprising 250 male and 250 female speakers are used for testing the proposed algorithm. Speakers have used a high quality directional microphone at a distance of about $5-10 \mathrm{cms}$ from their mouth, while uttering in a noiseless environment. Half of the data (125 males and 125 females) are used for training and the other half for testing. Speakers are made to utter the PSL primitives, which are recorded as reference words for later recognition. 


\section{Results and discussions}

The algorithm developed for recognition, as discussed in $\S 4$, is simulated using MATLAB version 5.3. The results of the test carried out are tabulated as shown in tables 2 and 3. In tables 2 and 3 results are shown only for some consonants with long vowels. Similarly, the experiment is conducted for all consonants with six long vowels and six short vowels (not shown in table) which have also shown the similar behaviour. The accuracy of the system is estimated by taking ten utterances of each speaker for each consonant and vowel combination, and for those ten utterances, the efficiency is calculated. Consonant-vowel recognition rates vary from $60 \%$ to $90 \%$ for male as well as female speakers.

The reduction of accuracy may be attributed to the speed of delivery, the pitch of the voice, and the pattern stressing. In addition, factors such as accents, age, regional dialects, speech impediments, gender, and emotional state also cause people to pronounce the $\mathrm{CV}$ combinations in different ways, as every individual speaker utterance is unique and also there is a strong dependence between recognition accuracy and word boundary determinations.

As the number of speakers used for training increases, the recognition efficiency also increases. Furthermore, it tends to a perfect speaker-dependent recognition system. Scope exists for improvement in recognition rates using better speech recognition techniques and better environment for recording the voices of the speakers. It is also observed that the accuracy of recognition also depends upon the clarity of pronunciation of the speakers.

Table 2. Accuracy of consonant-vowel (CV) recognition for male speaker.

\begin{tabular}{|c|c|c|c|c|c|c|c|c|c|c|c|}
\hline \multirow[b]{2}{*}{$\mathrm{CV}$} & \multirow{2}{*}{$\begin{array}{c}\text { PSL } \\
\text { symbol }\end{array}$} & \multicolumn{10}{|c|}{ Speakers $\%$ of recognition } \\
\hline & & 1 & 2 & 3 & 4 & 5 & 6 & 7 & 8 & 9 & 10 \\
\hline $\mathrm{Pa}$ & & 90 & 60 & 70 & 90 & 80 & 80 & 70 & 90 & 60 & 60 \\
\hline $\mathrm{Ba}$ & & 80 & 60 & 60 & 80 & 60 & 90 & 70 & 70 & 90 & 60 \\
\hline $\mathrm{Ta}$ & & 60 & 90 & 80 & 70 & 90 & 60 & 90 & 60 & 80 & 70 \\
\hline $\mathrm{Da}$ & & 80 & 90 & 60 & 90 & 90 & 70 & 80 & 80 & 90 & 90 \\
\hline $\mathrm{Ja}$ & & 70 & 80 & 70 & 90 & 90 & 80 & 80 & 90 & 90 & 90 \\
\hline $\mathrm{Ka}$ & & 60 & 90 & 90 & 70 & 90 & 60 & 90 & 60 & 90 & 80 \\
\hline $\mathrm{Ga}$ & $\bullet$ & 80 & 60 & 90 & 80 & 70 & 90 & 80 & 60 & 60 & 80 \\
\hline $\mathrm{Ma}$ & & 80 & 80 & 60 & 90 & 90 & 70 & 90 & 80 & 70 & 90 \\
\hline $\mathrm{Na}$ & & 90 & 90 & 90 & 80 & 90 & 90 & 90 & 80 & 90 & 90 \\
\hline $\mathrm{Sa}$ & ) & 90 & 60 & 60 & 60 & 90 & 80 & 70 & 90 & 90 & 80 \\
\hline
\end{tabular}


Table 3. Accuracy of consonant-vowel (CV) recognition for female speaker.

\begin{tabular}{|c|c|c|c|c|c|c|c|c|c|c|c|}
\hline \multirow[b]{2}{*}{$\mathrm{CV}$} & \multirow{2}{*}{$\begin{array}{c}\text { PSL } \\
\text { symbol }\end{array}$} & \multicolumn{10}{|c|}{ Speakers \% of recognition } \\
\hline & & 1 & 2 & 3 & 4 & 5 & 6 & 7 & 8 & 9 & 10 \\
\hline $\mathrm{Pa}$ & & 70 & 80 & 60 & 80 & 90 & 80 & 60 & 80 & 90 & 80 \\
\hline $\mathrm{Ba}$ & & 80 & 70 & 90 & 70 & 80 & 60 & 90 & 80 & 60 & 80 \\
\hline $\mathrm{Ta}$ & & 80 & 90 & 80 & 60 & 90 & 80 & 60 & 90 & 80 & 80 \\
\hline $\mathrm{Da}$ & & 60 & 90 & 90 & 70 & 80 & 80 & 80 & 60 & 80 & 80 \\
\hline $\mathrm{Ja}$ & & 60 & 80 & 80 & 60 & 90 & 80 & 60 & 80 & 60 & 80 \\
\hline $\mathrm{Ka}$ & & 80 & 70 & 90 & 60 & 90 & 70 & 80 & 90 & 80 & 80 \\
\hline $\mathrm{Ga}$ & $\overline{-}$ & 80 & 80 & 90 & 70 & 80 & 90 & 80 & 90 & 70 & 80 \\
\hline Ma & & 80 & 90 & 90 & 80 & 90 & 70 & 90 & 80 & 80 & 90 \\
\hline $\mathrm{Na}$ & & 90 & 80 & 80 & 80 & 90 & 70 & 90 & 80 & 80 & 90 \\
\hline $\mathrm{Sa}$ & ) & 80 & 80 & 90 & 60 & 80 & 80 & 70 & 90 & 80 & 90 \\
\hline
\end{tabular}

\section{Conclusion}

The work presented in this paper is an attempt towards automation of the process of transcribing the dictated/spoken text in English using PSL. The words formed out of combinations of consonants and vowels are considered. The developed algorithm is tested on varities of samples drawn from different Speakers. The recognition rate is quite satisfactory and the efficiency of recognition solely depends upon the pronunciation of speakers. Thus, scope exists for improvement in recognition rates using other techniques like neural networks, fuzzy logic etc. The ultimate aim of this area of research is the production of PSL text from spoken English text.

\section{References}

Davis K H, Biddulph R, Balashek S 1952 Automatic recognition of spoken digits. J. Acoust. Soc. Am. 24: 637-642

Pitman I 1976 Pitman shorthand instructor and key (Wheeler)

Rabiner L, Juang B-H 2003 Fundamentals of speech recognition. First Indian reprint (Pearson Education) pp 183-276

Rabiner L R, Levinson S E, Rosenberg A E, Wilpon J G 1979 Speaker independent recognition of isolated words using clustering techniques. IEEE Trans. Acoustics, Speech, Signal Process. ASSP27: 336-349

Vergin R, O’ Shaughnessy D, Farhat A 1999 Generalized Mel frequency cepstral coefficients for large-vocabulary speaker-independent continuous-speech recognition. IEEE Trans. Speech Audio Process. 7(5): 121-125 